\title{
ANALYSIS OF SENTINEL-1 DERIVED SOIL MOISTURE MAPS OVER OCCITANIE, SOUTH FRANCE
}

\author{
Nicolas Baghdadi ${ }^{1}$,Hassan Bazzi ${ }^{1}$, Mohammad El Hajj ${ }^{1}$, Mehrez Zribi ${ }^{2}$ \\ ${ }^{1}$ IRSTEA, UMR TETIS, University of Montpellier, 34093 Montpellier Cedex 5, France \\ ${ }^{2}$ CESBIO, CNRS, 31401 Toulouse cedex 9, France
}

\begin{abstract}
Monitoring the surface soil moisture (SSM) in agricultural areas at plot scale helps in many applications such as irrigation planning and crop management. Over the last decade, SAR (Synthetic Aperture Radar) data have shown great potential in estimating SSM over agriculture areas. Today, Sentinel-1 (S1) and Sentinel-2 (S2) satellites present a good opportunity for operational SSM estimates in agricultural areas because they provide free and open access data at high spatial resolution $(10 \mathrm{~m} \times 10 \mathrm{~m})$ and high revisit time (6 days over Europe). The aim of this paper is to present an operational approach for mapping soil moisture at high spatial resolution (plot scale) in agriculture areas by coupling $\mathrm{S} 1$ and S2 images. The proposed approach is based on the inversion of the Water Cloud Model (WCM) using the neural network technique.
\end{abstract}

Index Terms - Soil moisture, Sentinel-1, SAR, C-band, Neural Network.

\section{INTRODUCTION}

Soil moisture plays a key role in different hydrological processes (floods, runoff, evapotranspiration, infiltration, soil erosion, and imbalances in the water and carbon cycles). The arrival of Sentinel-1 SAR data (10 $\mathrm{m}$ spatial resolution and a 6-day revisit period) and Sentinel-2 optical data (10 m spatial resolution and a 5-day revisit period) have encouraged the development of operational algorithm for soil moisture mapping over agricultural areas with high revisit time and high spatial resolution (up to plot scale).

Several studies tend to use the neural networks technique to invert backscattering models and estimate the soil moisture. To estimate soil moisture and roughness from the C-band polarimetric radar data, Baghdadi et al. [1] first generated a database of backscattering coefficients for a wide range of bare soil conditions (soil moisture and surface roughness) using the Integral Equation Model IEM [2]. Then, the neural networks (NNs) were trained using the synthetic database. A priori information on the soil moisture and roughness were considered to improve the accuracy of neural networks. Finally, the trained neural networks were validated using a real database and show an accuracy on the soil moisture estimates of approximately $7 \mathrm{vol} \%$ with the use of a priori information. El Hajj et al. [3] inverted the Water Cloud Model (WCM) [4] combined with the Integral Equation model (IEM) [2,5]. In their study, they used the WCM parameterized by Baghdadi et al. [6] for the C-band. The total backscattering coefficient in the WCM is considered as the sum of the direct vegetation contribution and the soil contribution multiplied by the attenuation factor. Using a vegetation descriptor derived from optical images (Sentinel-2), the direct contribution of vegetation can be calculated. El Hajj et al. [3] validated the proposed approach for the operational mapping of soil moisture over a study site located in Occitanie, South France. They showed that the soil moisture in agricultural areas could be estimated with an accuracy of approximately 5 vol.\%. Moreover, an evaluation of SMOS, SMAP, ASCAT and S1-SSM products was performed by El Hajj et al. [7] and reveals that the S1-derived soil moisture maps provide the most accurate estimation of SSM. The higher accuracy of estimated SSM moisture is probably due to the well-calibrated IEM combined with the well parameterized WCM and the use of high spatial resolution $(10 \mathrm{~m} \times 10 \mathrm{~m})$ land cover maps derived from $\mathrm{S} 2$ images to eliminate SAR scattering from forest and urban areas.

The aim of this paper is to analyze Sentinel-1 derived soil moisture maps over Occitanie region according to GPM (Global Precipitation Mission) rainfall data. The soil moisture maps were derived from the very high spatial resolution Sentinel-1 images following the algorithm developed by El Hajj et al. [3]. The operational approach for mapping soil moisture at high spatial resolution (up to the plot scale) in agriculture areas by coupling S1 and S2 images is described in section 2. Section 3 includes a comparison between S1SSM and precipitation derived from GPM (Global Precipitation Measurement) data. Finally, Section 4 presents the main conclusions. 


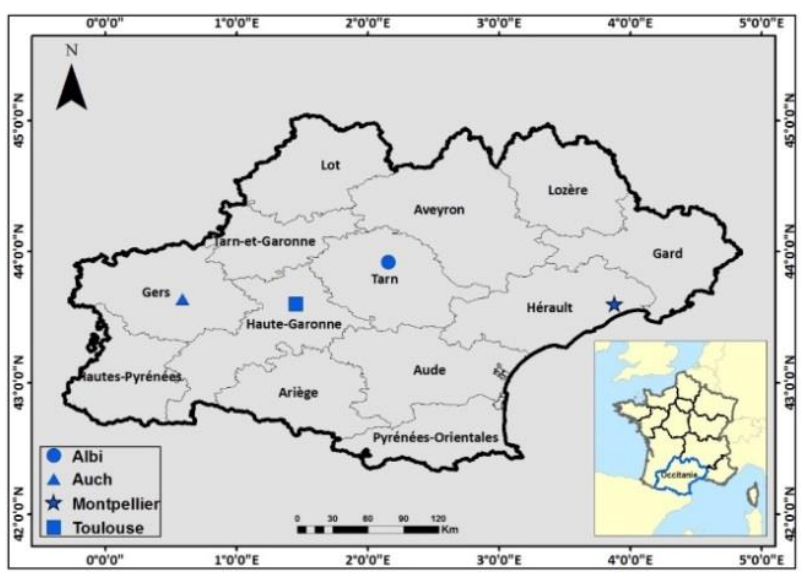

Figure 1. Location of study site, Occitanie region, South France.

\section{S1-SSM ALGORITHM}

The approach for the operational soil moisture mapping uses the neural networks $(\mathrm{NN})$ technique to invert the radar backscattering signal [3]. The development of the inversion method consists of the following steps:

- Generate a synthetic database of backscattering coefficients using a parameterized WCM [4] combined with the modified IEM [2,5];

- Noise the synthetic SAR C-band database and NDVI values to obtain synthetic database close to the real SAR and NDVI data;

- Train and validate the neural networks using the synthetic dataset;

- Finally, apply the trained neural networks to S1 and S2 images to map the soil moisture.

Incidence angle, noisy radar signal at $\mathrm{VV}$ polarization, and noisy NDVI are the inputs of the NN. The soil roughness (root mean square surface height "Hrms") was not used as an input parameter for the neural networks training and validation. However, the synthetic database was simulated using $H r m s$ values between 0.5 and $3.8 \mathrm{~cm}$. The $\mathrm{NN}$ was trained using the Levenberg-Marquardt algorithm with two hidden layers. The number of neurons for each hidden layer is 20. Linear and tangent-sigmoid transfer functions were associated with the first and second hidden layers, respectively.

To improve the soil moisture estimates, a priori knowledge on the soil moisture state is introduced. Indeed, from weather forecasts (precipitation and temperature) it is easy to define if the soil is is either dry to slightly wet (no precipitation for many days before SAR acquisition) or very wet (heavy rainfall preceding SAR acquisition). The integration of a priori information constrains the range of possible soil moisture parameter values estimated by the neural networks and thus leads to a better estimation of the soil moisture:

- Case 1: A priori information is available for $m v$. The soil is supposed to be dry to slightly wet according to expertise based mainly on meteorological data (precipitation, temperature). Soil moisture values are assumed to range from 2 to 25 vol. $\%$.

- Case 2: A priori information is available for $m v$. The soil is supposed to be very wet according to the expertise of the meteorological data. The $m v$ values are assumed to vary between 25 and 40 vol. \%.

An overlap of $10 \mathrm{vol} . \%$ on the $m v$ is considered between the databases used for the training of the neural networks. For a priori information that soil is dry to slightly wet (case 1), the NN was developed using the training database elements with the $m v$ between 2 and $30 \mathrm{vol}$. \%. For a priori information that soil is very wet (case 2), the $\mathrm{NN}$ was developed using the training database elements with the $m v$ between 20 and 40 vol. $\%$.

The developed algorithm is currently used in operational mode on many study sites. For example, S1 soil moisture maps for the Occitanie region (South France) at high spatial resolution (up to plot scale) are available since September 2016 as open access data via the Theia French Land Data Center (http://www.theia-land.fr/en/thematic-products).

\section{COMPARISON BETWEEN S1-SSM AND PRECIPITATION}

Rainfall measurements with 30 min revisit time obtained from the GPM (Global Precipitation Measurements) sensor were considered in this study. From the GPM mission the IMERG (Integrated Multi-satellite Retrievals for GPM) data product was selected to perform our analysis. The spatial resolution of IMERG maps is $0.1^{\circ} \times 0.1^{\circ}$. IMERG data at different spatial and temporal resolutions are available via the NASA Precipitation Measurements Mission (PMM) website (https://pmm.nasa.gov/data-access).

For each S1-SSM map date, one cumulative rainfall map for the 6 days prior to the S1-SSM map date was computed by summing the $30 \mathrm{~min}$ time interval of the Late IMERG GPM product. Then, each S1-derived SSM map was overlaid with the corresponding 6 days of cumulative GPM rainfall. Starting with the initial map on 21/12/2016 (Figure 2a), departments as Tarn, Hérault, Lozère, and Aveyron of the eastern and middle parts of the region encountered high soil moisture values (more than $20 \mathrm{vol} . \%$ ) corresponding to 10 $20 \mathrm{~mm}$ cumulative precipitation during the past 6 days, whereas Gers and Haute-Garonne of the western part shows low soil moisture values ( $20 \mathrm{vol} . \%$ at most) corresponding to the absence of precipitation in the area for the same prior period. A map derived 6 days later on 27/12/2016 (Figure 2b) shows a decrease in soil moisture values in the Hérault and Gard due to the absence of precipitation between 21/12/2016 and 27/12/2016. Moreover, high soil moisture values are estimated for Tarn due to continuous raining episodes 
between 21/12/2017 and 27/12/2017, and a slight increase in soil moisture values is observed in western part due to $3 \mathrm{~mm}$ of precipitation recorded one day prior to the S1-SSM map according to the GPM data (Figure 2b). A general decrease in soil moisture values throughout the region is observed between 27/12/2016 (Figure 2b) and 02/01/2017 (Figure 2c) as no precipitation was recorded. A continuous lack of precipitation for 12 days from 27/12/2016 until 08/01/2017 caused soil moisture values to drop to less than $15 \mathrm{vol} . \%$ on $08 / 01 / 2017$ in the whole region as shown in Figure 2d. Six days later on 14/01/2017 (Figure 2e), soil moisture values remained low at the east as no precipitation was recorded for an additional 6 days, whereas a moderate increase in soil moisture values is observed in the middle and western parts (Tarn, Haute-Garonne, and Gers) corresponding to precipitation between $08 / 01 / 2017$ and 14/01/2017 (a cumulative rainfall increase of 5-20 mm). Twenty-four days with no precipitation recorded in the Hérault and Gard region between 27/12/2016 and 20/01/2017 caused the soil moisture values to drop more, with values less than 15 vol.\% in this area on 20/01/2017 (Figure 2f).

In addition, for Gers department, low soil moisture values (less than 15 vol.\%) were estimated on January 20, 2017 and high cumulative precipitation was recorded between 14 and 20 January 2017 (15 mm) (Figure 2f). This low soil moisture estimates could be linked to the presence of frozen soil in the area. In a recent study using Sentinel-1 SAR data, Baghdadi et al. [8] showed that a decrease of at least 3 $\mathrm{dB}$ in the radar backscattered signal can be observed over frozen soil conditions. Such a decrease in the SAR signal yields an underestimation of soil moisture in the S1-derived SSM maps. In our case, it seems that low soil moisture values for this area on $20 / 01 / 2017$, although with the presence of rainfall, is due to frozen soil conditions. To support our assumption, the temperature curve and precipitation records for a local meteorological station in Auch city located in Gers-Occitanie were analyzed. On 19 January 2017 (S1 acquisition over Gers on January 19, 2017 17:56 UTC), the temperature varied between $-8^{\circ} \mathrm{C}$ and $2^{\circ} \mathrm{C}$ with a mean value of $-3^{\circ} \mathrm{C}$ throughout the day. Three days before $(15$ and 16 January) $15 \mathrm{~mm}$ cumulative precipitation was recorded. Thus, a precipitation event followed by a decrease in the air temperature to less than $0^{\circ} \mathrm{C}$ justifies the presence of frozen soil conditions as the cause of a decrease in the estimated soil moisture values in the S1-derived SSM maps.

After 29 days of dry conditions in the eastern part (Hérault and Gard between 27/12/2016 and 25/01/2017), a sudden increase in the soil moisture was observed on 26/01/2017 with the beginning of a rainfall event (Figure 2g). The mean S1-SSM mean value in this area increased from 13 vol. $\%$ in $20 / 01 / 2017$ to 32 vol. $\%$ in $26 / 01 / 2017$. On the other hand, the absence of precipitation in the remaining parts of the region between 20/01/2017 and 26/01/2017 caused soil moisture to attain lower values ( 16 vol. $\%$ ) on $26 / 01 / 2017$. The slight increase in soil moisture values in Gers between 20 and 26 January is probably due to the disappearance of the frozen conditions observed in the previous acquisition (20/01/2017). The temporal analysis of S1-derived SSM values over a period of time shows the direct effect of raining episodes or dry conditions on S1-SSM values.
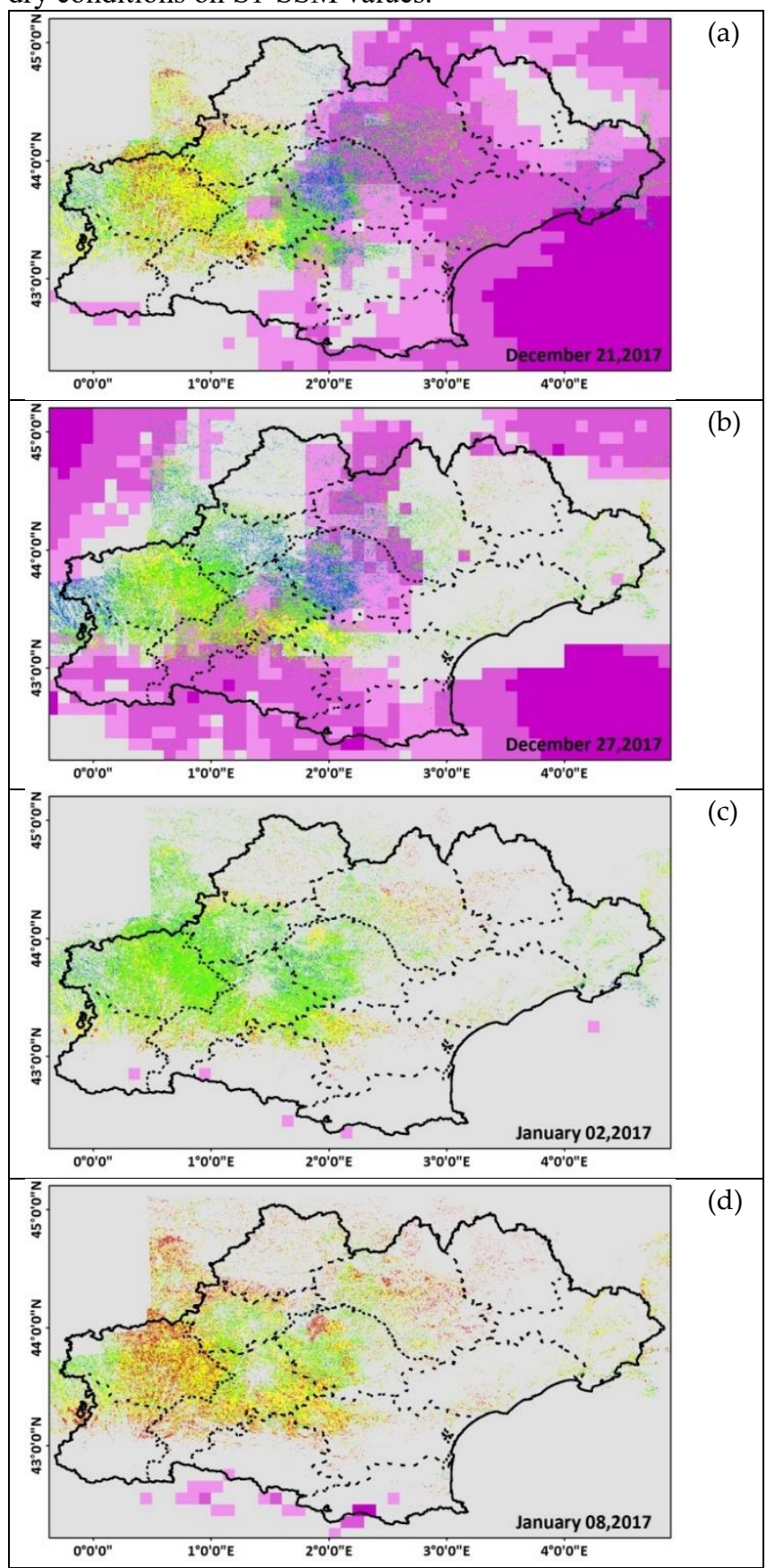


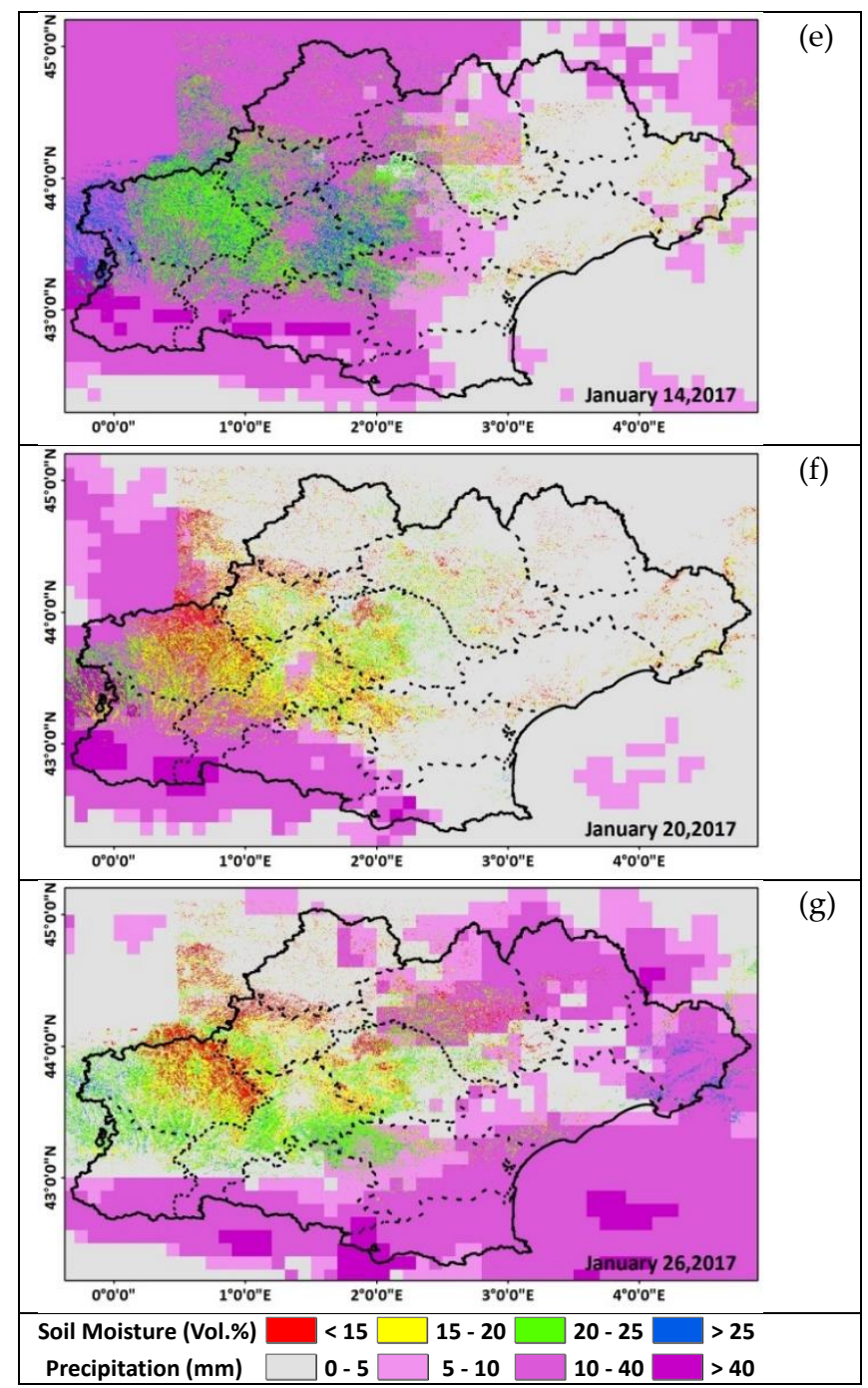

Figure 2: S1-derived SSM map overlaid with GPM cumulative precipitation data. (a) $21 / 12 / 2016$, (b) $27 / 12 / 2016$, (c) $02 / 01 / 2017$, (d) $08 / 01 / 2017$, (e) 14/01)2017, (f) 20/01/2017, (g) 26/01/2017.

\section{CONCLUSION}

This paper presents an analysis of the new Sentinel-1 surface soil moisture (S1-SSM) product derived at a very high spatial resolution (plot scale) and a revisit time of 6 days over Southern of France. A good coherence was observed between the temporal evolution of the soil moisture and the precipitation records derived from the GPM data. The S1SSM values increases following rainfall event and decreases after a period without rainfall due to evaporation.

\section{ACKNOWLEDGMENT}

This research was supported by IRSTEA (National Research Institute of Science and Technology for Environment and Agriculture) and the French Space Study Center (CNES, DAR 2018 TOSCA). The authors wish to thank the ESA
(European Commission and the European Space Agency) for providing the S1 images. We used Copernicus level 2A S2 data processed by the French land data service center (Theia: https://www.theia-land.fr).

\section{REFERENCES}

[1] N. Baghdadi, R. Cresson, M. El Hajj, R. Ludwig, and I. La Jeunesse, "Estimation of soil parameters over bare agriculture areas from C-band polarimetric SAR data using neural networks.," Hydrol. Earth Syst. Sci., vol. 16, no. 6, pp. 1607-1621, 2012.

[2] N. Baghdadi, N. Holah, and M. Zribi, "Calibration of the integral equation model for SAR data in C-band and $\mathrm{HH}$ and VV polarizations," Int. J. Remote Sens., vol. 27, no. 4, pp. 805-816, 2006.

[3] El Hajj, M.; Baghdadi, N.; Zribi, M.; Bazzi, H. Synergic use of Sentinel-1 and Sentinel-2 images for operational soil moisture mapping at high spatial resolution over agricultural areas. Remote Sens. 2017, 9, 1292, doi:10.3390/rs9121292.

[4] Attema, E.P.W.; Ulaby, F.T. Vegetation modeled as a water cloud. Radio Science 1978, 13, 357-364, doi:10.1029/RS013i002p00357.

[5] Baghdadi, N.; Abou Chaaya, J.; Zribi, M. Semiempirical Calibration of the Integral Equation Model for SAR Data in C-Band and Cross Polarization Using Radar Images and Field Measurements. IEEE Geoscience and Remote Sensing Letters 2011, 8, 14-18, doi:10.1109/LGRS.2010.2050054.

[6] Baghdadi, N.; El Hajj, M.; Zribi, M.; Bousbih, S. Calibration of the Water Cloud Model at C-Band for Winter Crop Fields and Grasslands. Remote Sensing 2017, 9, 969, doi:10.3390/rs9090969.

[7] El Hajj, M.; Baghdadi, N.; Zribi, M.; RodríguezFernández, N.; Wigneron, J.; Al-Yaari, A.; Al Bitar, A.; Albergel, C.; Calvet, J.-C. Evaluation of SMOS, SMAP, ASCAT and Sentinel-1 Soil Moisture Products at Sites in Southwestern France. Remote Sensing 2018, 10, 569, doi:10.3390/rs10040569.

[8] Baghdadi, N.; Bazzi, H.; El Hajj, M.; Zribi, M. Detection of Frozen Soil Using Sentinel-1 SAR Data. Remote Sensing 2018, 10, 1182, doi:10.3390/rs10081182. 